\title{
A Two Parameter Odd Exponentiated Skew-T Distribution With J-Shaped Hazard Rate Function
}

\author{
Obinna Damian Adubisi ${ }^{1 *}$, Ahmed Abdulkadir ${ }^{2}$, Haruna Chiroma ${ }^{3}$ \\ 1. Department of Mathematics and Statistics, Federal University Wukari, Taraba State, Nigeria. \\ ${ }^{1,2,3 .}$ Department of Mathematical Sciences, Abubakar Tafawa Balewa University, Bauchi State,
} Nigeria.

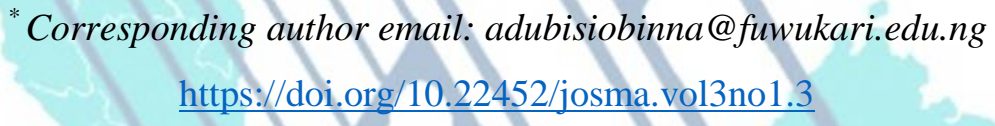

A new generalization of the skew-t distribution was proposed. The two-parameter lifetime model called the odd exponentiated skew-t distribution has the ability of fitting skewed, long and heavy tailed datasets. It is considered to be more flexible than the skew-t distribution as it contains it as a special case. Some basic properties of the distribution such as the order statistics, entropy, asymptotic behaviour, moment, incomplete moment, characteristic function and quantile function were derived. The odd exponentiated skew-t distribution parameter estimates were derived using the maximum likelihood estimation method and simulation studies performed to evaluate the finite sample performance of these parameter estimates showed that the parameter estimates were consistent and approached the arbitrary selected parameter values as the sample size is increased. The application using a real-life dataset indicated that the new distribution outperformed the other competing distributions. The hazard rate shape of the odd exponentiated skew-t distribution was found to be increasing and $\mathrm{J}$-shaped which was also reflected in the application result.

Keywords: Maximum likelihood estimation, Moments, Order statistics, Skew-t distribution, Odd exponentiated generator.

\section{INTRODUCTION}

The methods of extending the flexibility of various continuous probability distributions are wellknown in the literature. Hence, significant efforts in developing new families of flexible continuous probability distributions and extending the efficacy of the existing distributions have been made by several authors over the years due to inability of the classical distributions to fit various real-life datasets. The skew-t distribution introduced as an extension of the symmetric t-distribution has been 
used extensively especially in the field of econometric, time series and financial analysis. Numerous authors have introduced various complex forms of the skew-t which lacked a defined expression for the density function, example of the forms can be found in Johnson et al. (1995), Azzalini and Capitanio (2003), Sahu et al. (2003) and Gupta (2002). Several authors have studied possible extensions and generalizations of the skew-t distribution: Shafiei and Doostparast (2014) proposed a new generalization of the skew-t distribution of Azzalini and Capitanio (2003) called the Balakrishnan skew-t (BST) distribution, as a scale mixture of the Balakrishnan normal distribution. The density function shape of the BST is right-skewed at different degree of freedom which gives it more flexibility in fitting skewed datasets. Aas and Haff (2006) proposed the generalized hyperbolic skew-t (GHST) distribution which is considered as a limiting case of the generalized hyperbolic (GH) distribution. They stated that the generalized hyperbolic skew-t (GHST) distribution can be represented as a mixing distribution comprising normal variance-mean mixture with the generalized inverse gaussian distribution. Khamis et al. (2017) proposed the Kumaraswamy skew-t (KwST) distribution which has the ability of fitting heavy-tailed and skewed datasets than the skew-t distribution of Azzalini and Capitanio (2014). Basalamah et al. (2018) introduced a new generalization of the skew-t distribution of Azzalini and Capitanio (2014) called the Beta skew-t (BST) distribution. The maximum likelihood and L-moments methods were used in demonstrating the flexibility of the BST distribution in fitting real datasets and the results were in favour of the BST distribution. These presented extensions with a lot of parameters were based on the complex skew-t distribution.

The noncomplex one-parameter tractable skew-t distribution introduced by Jones and Faddy (2003) with defined density and distribution functions was established by introducing a scaling factor into the two degrees of freedom of the simplest student-t form derived by Jones (2002). The main aim of this article is to introduce a new hybridized distribution with fewer parameters, with the expectation it produces a better fit in certain real-world situations and in a wider range of real-life datasets in engineering, biology, medicine and finance. Additionally, a complete derivation of the statistical properties of the proposed distribution are provided. The purpose for developing the two-parameter hybridized distribution is to furnish a more flexible distribution with skewed and unimodal features that can handle properly skewed and leptokurtic real datasets often found in various fields better than existing two-parameter distributions. This new distribution in this article can find its potentiality as an alternative conditional error distribution in GARCH framework when used in volatility modeling. The rest of the paper is organized as follows. In Section 2, introduce the new distribution called the odd exponentiated skew-t $\left(\mathrm{OE}_{\mathrm{ST}}\right)$ distribution. In Section 3, statistical properties of the proposed distribution are derived. In section 4, the maximum likelihood estimation method is applied to derive the estimates of the model parameters and simulation study performed to assess the performance of 
the $\mathrm{OE}_{\mathrm{ST}}$ parameter estimates. In section 5, a dataset application is illustrated to demonstrate the superiority of the new distribution while section 6 concludes the study.

\section{ODD EXPONENTIATED SKEW-T DISTRIBUTION}

Jones (2001) and Jones and Faddy (2003) established a tractable skewed extension of the symmetric student-t distribution known as the skew student-t (skew-t) distribution. The cumulative distribution function $(\mathrm{CDF})$ is given as:

$G_{S T}(y)=\frac{1}{2}\left(1+\frac{y}{\sqrt{\lambda+y^{2}}}\right), \quad y \in(-\infty, \infty)$

The probability distribution function (PDF) obtained by differentiating (1) is given as

$$
g_{S T}(y)=\frac{\lambda}{2\left(\lambda+y^{2}\right)^{3 / 2}}
$$

where $\lambda$ is the skew parameter.

The odd exponentiated family of distributions is a special case established by setting $\beta=1$ in the density and distribution functions of the Weibull-G family (Bourguignon et al., 2014). The CDF is given by

$$
F(y)=\left\{1-e^{-\alpha \frac{G(y)}{1-G(y)}}\right\},
$$

The PDF by differentiating (3) is given as:

$$
f(y)=\frac{\alpha g(y)}{[1-G(y)]^{2}} e^{-\alpha \frac{G(y)}{1-G(y)}},
$$

where $\alpha>0$ is the shape parameter, $G(y)$ and $g(y)$ are the baseline distribution CDF and PDF. A two-parameter model called the odd exponentiated skew-t $\left(\mathrm{OE}_{\mathrm{ST}}\right)$ distribution is introduced. The PDF is obtained by inserting Equations (1) and (2) into Equation (4) expressed as: 


$$
f(y ; v)=\frac{\alpha \lambda}{2\left(\lambda+y^{2}\right)^{3 / 2}\left[1-\left(\frac{1}{2}\left(1+\frac{y}{\sqrt{\lambda+y^{2}}}\right)\right)\right]^{2}} e^{-\frac{\left(\frac{1}{2}\left(1+\frac{y}{\sqrt{\lambda+y^{2}}}\right)\right)}{1-\left(\frac{1}{2}\left(1+\frac{y}{\sqrt{\lambda+y^{2}}}\right)\right)}}, \quad y \in(-\infty, \infty) ; \alpha, \lambda>0,
$$

The corresponding CDF by inserting (1) in (3) is given as:

$F(y ; \nu)=1-e^{-\alpha \frac{\left(\frac{1}{2}\left(1+\frac{y}{\sqrt{\lambda+y^{2}}}\right)\right)}{1-\left(\frac{1}{2}\left(1+\frac{y}{\sqrt{\lambda+y^{2}}}\right)\right)}}$

From now onward, a random variable $Y$ having PDF (5) is denoted by $O E_{S T}(v)$, where $v=(\alpha, \lambda)$ are set of parameters.

The survival function is defined as $s(y)=1-F(y)$, given a random variable $Y$. Hence, the survival function $s(y)$ of $\mathrm{OE}_{\mathrm{ST}}$ distribution is given as:

$S(y)=e^{-\alpha \frac{\left(\frac{1}{2}\left(1+\frac{y}{\sqrt{\lambda+y^{2}}}\right)\right)}{1-\left(\frac{1}{2}\left(1+\frac{y}{\sqrt{\lambda+y^{2}}}\right)\right)}}$

The hazard rate function $h(y)$ is given as:

$$
h(y)=\frac{\alpha \lambda}{2\left(\lambda+y^{2}\right)^{3 / 2}\left[1-\left(\frac{1}{2}\left(1+\frac{y}{\sqrt{\lambda+y^{2}}}\right)\right)\right]^{2}}
$$

To show the efficacy of the $\mathrm{OE}_{\mathrm{ST}}$ distribution, the graphical structures of the $\mathrm{OE}_{\mathrm{ST}}$ density function and distribution function are depicted in Figures 1 and 2 with the skew parameter $\lambda$ kept constant and the shape parameter $\alpha$ varied. Figure 1, indicates that the right tail of the density function gets lighter and tend to zero as $\alpha$ approach infinity. More so, Figure 2 indicates that the shape of the CDF is within the limits of zero and one, which justifies that the proposed $\mathrm{OE}_{S \mathrm{ST}}$ distribution is a valid distribution. 

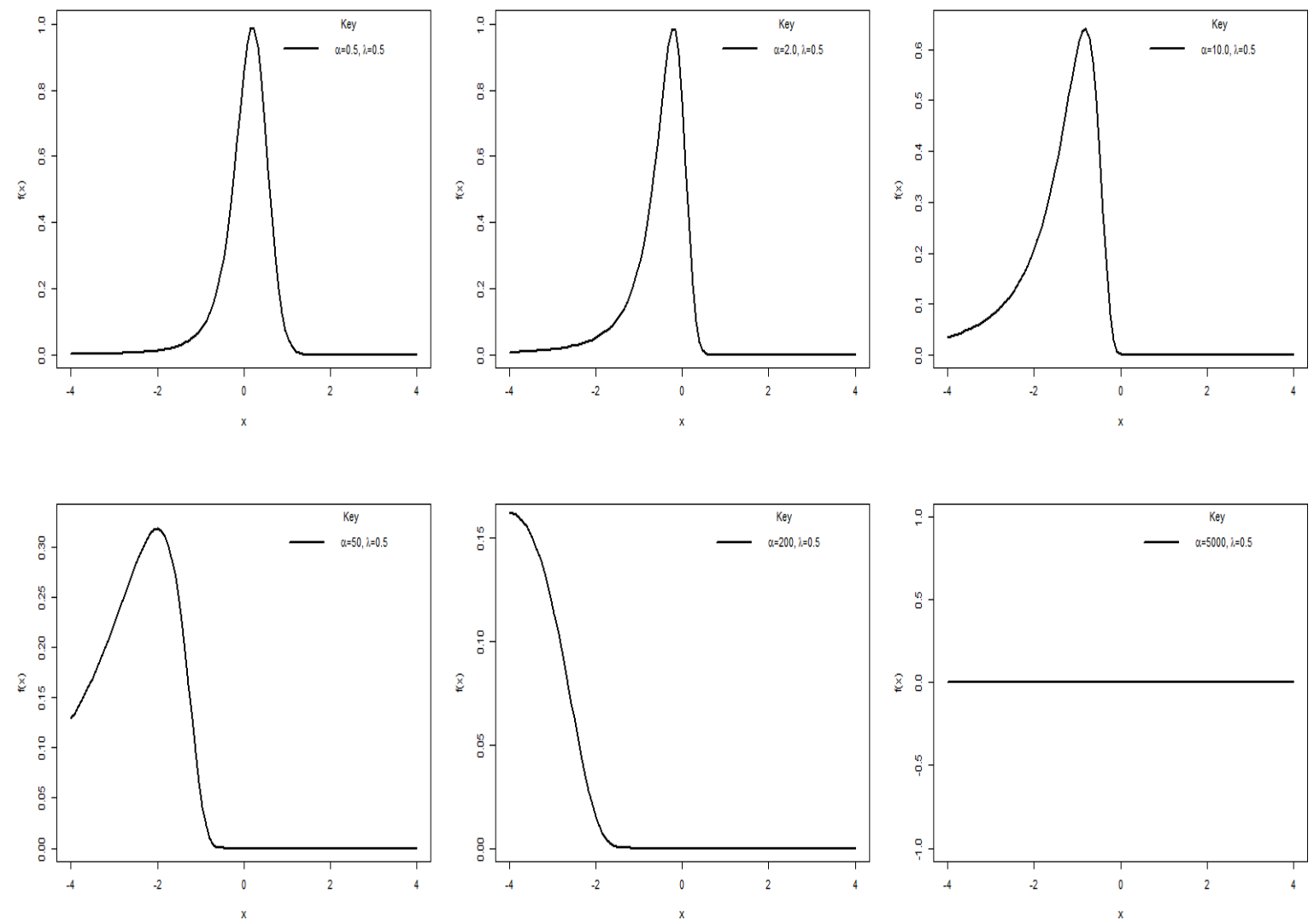

Figure 1: The $\mathrm{OE}_{\mathrm{ST}}$ density function plots for some selected $(\alpha=$ varied, $\lambda=0.5)$ parameter values.
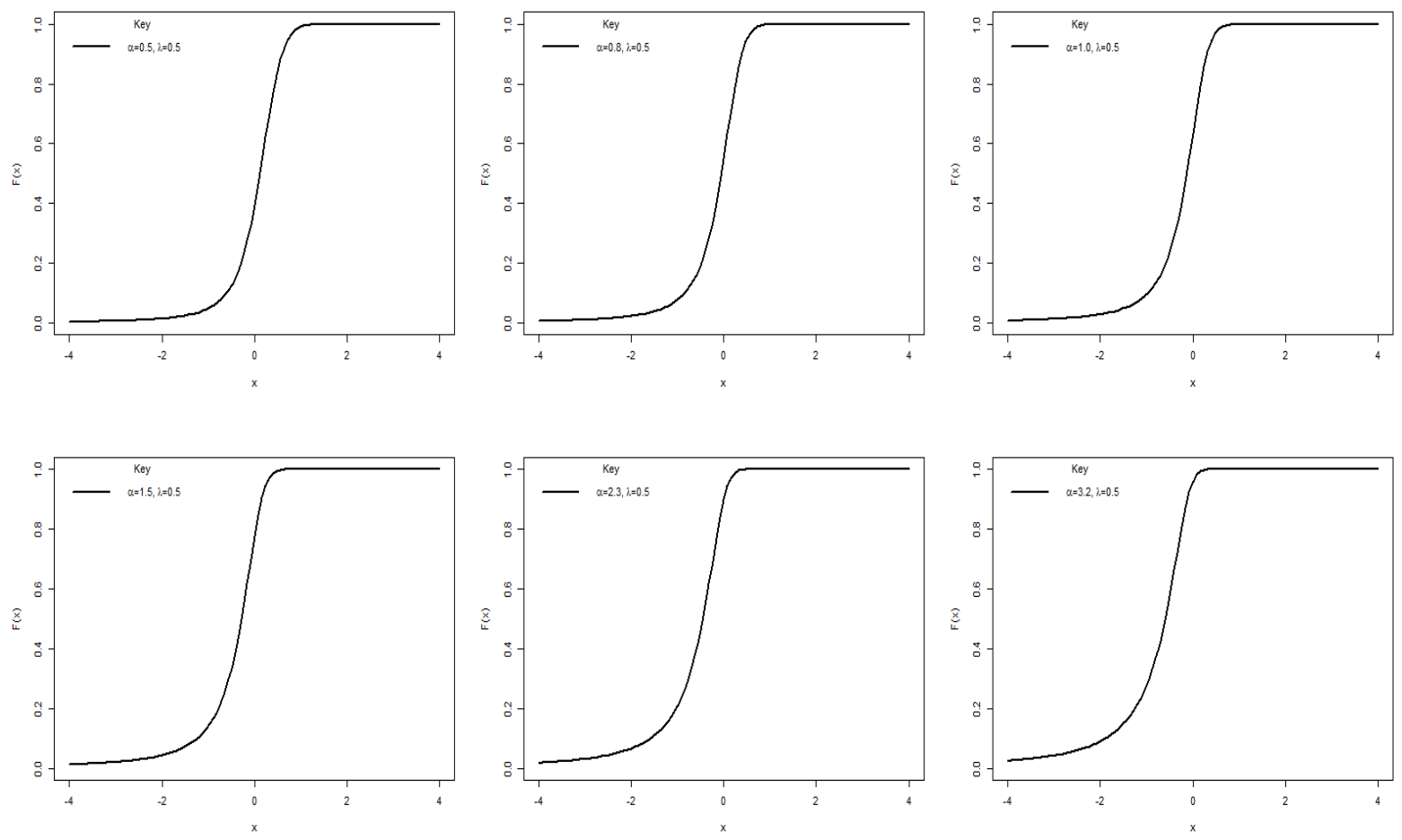

Figure 2: The $\mathrm{OE}_{\mathrm{ST}}$ distribution function plots for some selected $(\alpha=$ varied, $\lambda=0.5)$ parameter values. 
Likewise, the shapes of the hazard rate function depicted in Figure 3, reveal that it can be increasing and J-shaped.
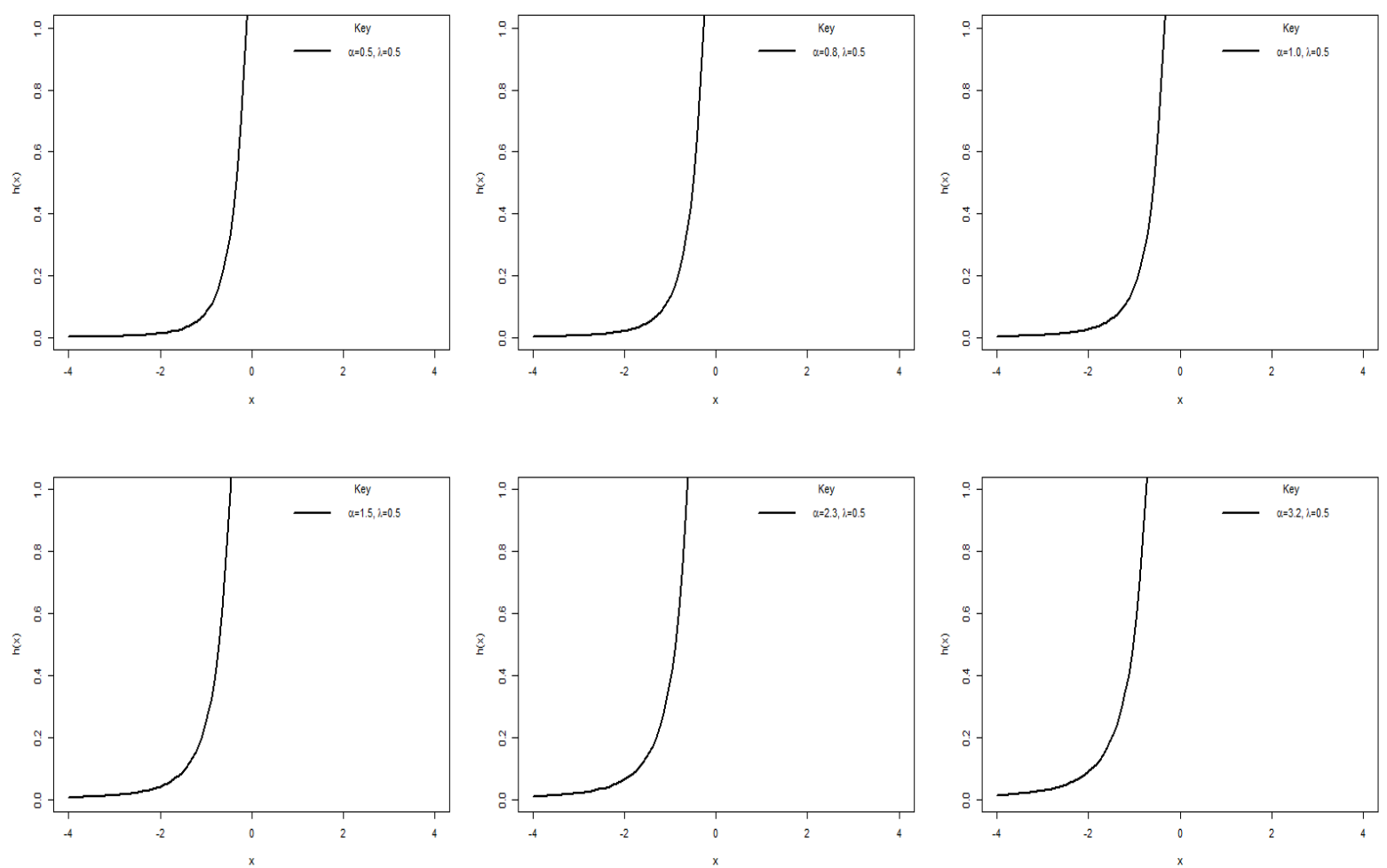

Figure 3: The $\mathrm{OE}_{\mathrm{ST}}$ hazard rate function plots for some selected $(\alpha=$ varied, $\lambda=0.5)$ parameter values.

\section{STATISTICAL PROPERTIES}

In this section, some basic statistical properties of the $\mathrm{OE}_{\mathrm{ST}}$ distribution are derived.

\subsection{Quantile Function}

The quantile function $Q(u)=F(y)^{-1}$ for $u \in(0,1)$ of the $\mathrm{OE}_{\mathrm{ST}}$ distribution is given by:

$$
Q(u)=\frac{\lambda^{1 / 2}\left(2\left\langle\frac{-\log (1-u)}{(\alpha-\log (1-u))}\right\rangle-1\right)}{\left(1-\left(2\left\langle\frac{-\log (1-u)}{(\alpha-\log (1-u))}\right\rangle-1\right)^{2}\right)^{1 / 2}}, \quad u \in(0,1) .
$$

The median $Q(0.5)$ is derived by setting $u=0.5$ in (9). The other quantiles can be derived similarly by setting $u=0.25$ and $u=0.75$. 


$$
Q(0.5)=\frac{\lambda^{1 / 2}\left(2\left\langle\frac{-\log (0.5)}{(\alpha-\log (0.5))}\right\rangle-1\right)}{\left(1-\left(2\left\langle\frac{-\log (0.5)}{(\alpha-\log (0.5))}\right\rangle-1\right)^{2}\right)^{1 / 2}}, \quad u \in(0,1)
$$

The $\mathrm{OE}_{\mathrm{ST}}$ quantile function (9) can be used in generating random values from the $\mathrm{OE}_{\mathrm{ST}}$ distribution. The Bowley skewness (Kenney \& Keeping,1962) and Moors kurtosis (Moors, 1988) are as follows:

$$
\begin{aligned}
& S_{k}=\frac{Q\left(\frac{3}{4} ; \varphi, \lambda\right)-2 Q\left(\frac{1}{2} ; \varphi, \lambda\right)+Q\left(\frac{1}{4} ; \varphi, \lambda\right)}{Q\left(\frac{3}{4} ; \varphi, \lambda\right)-Q\left(\frac{1}{4} ; \varphi, \lambda\right)} \\
& K=\frac{Q\left(\frac{7}{8} ; \varphi, \lambda\right)-Q\left(\frac{5}{8} ; \varphi, \lambda\right)-Q\left(\frac{3}{8} ; \varphi, \lambda\right)+Q\left(\frac{1}{8} ; \varphi, \lambda\right)}{Q\left(\frac{6}{8} ; \varphi, \lambda\right)-Q\left(\frac{2}{8} ; \varphi, \lambda\right)}
\end{aligned}
$$

where $Q($.$) represent the quantile function. Using the \mathrm{OE}_{\mathrm{ST}}$ quantile function (9), the numeric values of the median (M), $25^{\text {th }}$ and $75^{\text {th }}$ percentiles, interquartile range (IQR), kurtosis (Ks), and skewness (Sk) for some chosen parameter values are provided in Table 1. It is clear from Table 1, as the values of $\lambda$ increases at specific values of $\alpha$, the skewness and kurtosis remain constant. More so, across different values of $\alpha$, the skewness and kurtosis decreases indicating negative properties, respectively.

Table 1: Descriptive statistics of the $\mathrm{OE}_{\mathrm{ST}}$ distribution.

\begin{tabular}{|l|l|l|l|l|l|l|l|}
\hline$\alpha$ & $\lambda$ & $\mathbf{K s}$ & $\mathbf{S k}$ & $\mathbf{M}$ & $\mathbf{2 5}^{\text {th }}$ & $\mathbf{7 5}^{\text {th }}$ & IQR \\
\hline $\mathbf{0 . 2}$ & 0.3 & -0.0511 & -0.0161 & 0.3627 & 0.1001 & 0.6170 & 0.5169 \\
\hline & 0.5 & -0.0511 & -0.0161 & 0.4683 & 0.1292 & 0.7965 & 0.6673 \\
\hline & 0.9 & -0.0511 & -0.0161 & 0.6283 & 0.1734 & 1.0687 & 0.8953 \\
\hline & 1.2 & -0.0511 & -0.0161 & 0.7254 & 0.2002 & 1.2340 & 1.0338 \\
\hline & 1.5 & -0.0511 & -0.0161 & 0.8111 & 0.2238 & 1.3796 & 1.1558 \\
\hline $\mathbf{0 . 4}$ & 0.3 & -0.1616 & -0.0725 & 0.1525 & -0.0907 & 0.3627 & 0.4534 \\
\hline & 0.5 & -0.1616 & -0.0725 & 0.1968 & -0.1171 & 0.4683 & 0.5853 \\
\hline & 0.9 & -0.1616 & -0.0725 & 0.2641 & -0.1571 & 0.6283 & 0.7853 \\
\hline & 1.2 & -0.1616 & -0.0725 & 0.3049 & -0.1813 & 0.7254 & 0.9068 \\
\hline & 1.5 & -0.1616 & -0.0725 & 0.3409 & -0.2028 & 0.8111 & 1.0138 \\
\hline $\mathbf{0 . 6}$ & 0.3 & -0.2291 & -0.1106 & 0.0396 & -0.2059 & 0.2361 & 0.4420 \\
\hline
\end{tabular}




\begin{tabular}{|l|l|l|l|l|l|l|l|}
\hline & 0.5 & -0.2291 & -0.1106 & 0.0511 & -0.2658 & 0.3048 & 0.5706 \\
\hline & 0.9 & -0.2291 & -0.1106 & 0.0685 & -0.3658 & 0.4089 & 0.7655 \\
\hline & 1.2 & -0.2291 & -0.1106 & 0.0791 & -0.4117 & 0.4722 & 0.8840 \\
\hline & 1.5 & -0.2291 & -0.1106 & 0.0884 & -0.4603 & 0.5280 & 0.9883 \\
\hline $\mathbf{0 . 7}$ & 0.3 & -0.2539 & -0.1253 & -0.0027 & -0.2516 & 0.1908 & 0.4424 \\
\hline & 0.5 & -0.2539 & -0.1253 & -0.0035 & -0.3248 & 0.2463 & 0.5712 \\
\hline & 0.9 & -0.2539 & -0.1253 & -0.0047 & -0.4358 & 0.3305 & 0.7663 \\
\hline & 1.2 & -0.2539 & -0.1253 & -0.0054 & -0.5032 & 0.3816 & 0.8848 \\
\hline & 1.5 & -0.2539 & -0.1253 & -0.0060 & -0.5626 & 0.4266 & 0.9892 \\
\hline $\mathbf{1 . 5}$ & 0.3 & -0.3599 & -0.1935 & -0.2167 & -0.5054 & -0.0216 & 0.4838 \\
\hline & 0.5 & -0.3599 & -0.1935 & -0.2797 & -0.6525 & -0.0279 & 0.6246 \\
\hline & 0.9 & -0.3599 & -0.1935 & -0.3753 & -0.8754 & -0.0374 & 0.8380 \\
\hline & 1.2 & -0.3599 & -0.1935 & -0.4334 & -1.0108 & -0.0432 & 0.9676 \\
\hline & 1.5 & -0.3599 & -0.1935 & -0.4846 & -1.1301 & -0.0483 & 1.0818 \\
\hline
\end{tabular}

\subsection{Asymptotic Behaviour}

The limits of the $\mathrm{OE}_{\mathrm{ST}}$ density function (PDF) are given by:

$\lim _{y \rightarrow-\infty} f(y)=\lim _{y \rightarrow+\infty} f(y)=0$

Proof:

For $y \rightarrow-\infty$, we have

$\lim _{y \rightarrow-\infty} f(x)=\lim _{y \rightarrow-\infty}\left(\frac{\alpha \lambda}{2\left(\lambda+y^{2}\right)^{3 / 2}\left[1-\left(\frac{1}{2}\left(1+\frac{y}{\sqrt{\lambda+y^{2}}}\right)\right)\right]^{2}} e^{\left.-\alpha \frac{\left(\frac{1}{2}\left(1+\frac{y}{\sqrt{\lambda+y^{2}}}\right)\right.}{\left.1-\left(1+\frac{y}{\sqrt{\lambda+y^{2}}}\right)\right)}\right)}\right)$

It is obvious that $\lim _{x \rightarrow-\infty}\left(\frac{\lambda}{2\left(\lambda+x^{2}\right)^{3 / 2}}\right)=0$.

Therefore, $\lim _{y \rightarrow-\infty} f(x)=0$

Similarly, for $y \rightarrow+\infty$, we have 


$$
\lim _{y \rightarrow+\infty} f(x)=\lim _{y \rightarrow+\infty}\left(\frac{\alpha \lambda}{2\left(\lambda+y^{2}\right)^{3 / 2}\left[1-\left(\frac{1}{2}\left(1+\frac{y}{\sqrt{\lambda+y^{2}}}\right)\right)\right]^{2}} e^{-\alpha \frac{\left(\frac{1}{2}\left(1+\frac{y}{\sqrt{\lambda+y^{2}}}\right)\right)}{1-\left(\frac{1}{2}\left(1+\frac{y}{\sqrt{\lambda+y^{2}}}\right)\right)}}\right)
$$

It is obvious that $\lim _{x \rightarrow+\infty}\left(\frac{\lambda}{2\left(\lambda+x^{2}\right)^{3 / 2}}\right)=0$.

Therefore, $\lim _{y \rightarrow+\infty} f(x)=0$

The results of the asymptotic behaviour infer that the $\mathrm{OE}_{S \mathrm{ST}}$ mode is unique.

\subsection{Mixture Representations}

The series expansion of the $\mathrm{OE}_{\mathrm{ST}}$ distribution is derived for the density and cumulative functions. This mixture representation is important to derive several statistical properties of this distribution in full generality. If $|s|<1$ and $k$ a positive real non-integer, the generalized binomial theorem representation is given by:

$(1-s)^{k-1}=\sum_{j=0}^{\infty}(-1)^{j}\left(\begin{array}{c}k-1 \\ j\end{array}\right) s^{j}$

The expanded form of the PDF, applying the series expansion in Equation (17) in (5) leads to:

$$
f(y)=\alpha \sum_{i, j=0}^{\infty} \frac{(-1)^{i+j} \alpha^{i}}{i !}\left(\begin{array}{c}
-(i+2) \\
j
\end{array}\right)\left(\frac{\lambda}{2\left(\lambda+y^{2}\right)^{3 / 2}}\right)\left[\frac{1}{2}\left(1+\frac{y}{\sqrt{\lambda+y^{2}}}\right)\right]^{(i+j)}
$$

The preceding equation reveals that the PDF expression is likely an infinite linear combination of the skew-t density functions. Thus, we can obtain the statistical properties of the $\mathrm{OE}_{\mathrm{ST}}$ distribution from the properties of the skew-t distribution. Also, another expanded form of the PDF is given by

$f(y)=w_{i, j, k, l, m} y^{m}\left(\lambda+y^{2}\right)^{-\left(\frac{m+3}{2}\right)}$

where $w_{i, j, k, l, m}=\alpha \lambda \frac{1}{2^{l+1}} \sum_{i, j, k, l=0}^{\infty} \sum_{m=0}^{l} \frac{(-1)^{i+j+k+l} \alpha^{i}}{i !}\left(\begin{array}{c}-(i+2) \\ j\end{array}\right)\left(\begin{array}{c}i+j \\ l\end{array}\right)\left(\begin{array}{c}k \\ l\end{array}\right)\left(\begin{array}{c}l \\ m\end{array}\right)$.

The expanded form of the CDF of the $\mathrm{OE}_{\mathrm{ST}}$ distribution by applying series expansion to Equation (6), is given by

$$
F(y)=\psi_{i, j, k, l, m, q} x^{q}\left(\lambda+y^{2}\right)^{-\frac{q}{2}}
$$


where $\psi_{i, j, k, l, m, q}=\frac{1}{2^{m}} \sum_{i, j, k, l, m=0}^{\infty} \sum_{q=0}^{m} \frac{(-1)^{i+j+k+l+m}(i \alpha)^{j}}{j !}\left(\begin{array}{c}j \\ k\end{array}\right)\left(\begin{array}{c}j+k \\ l\end{array}\right)\left(\begin{array}{c}l \\ m\end{array}\right)\left(\begin{array}{c}m \\ q\end{array}\right)$

\subsection{Moments}

Let $Y \sim O E_{S T}(\alpha, \lambda)$ be a random variable, then the $\mathrm{g}^{\text {th }}$ moment of $Y$ is given by

$$
\mu_{g}^{\prime}=\int_{-\infty}^{+\infty} y^{g} w_{i, j, k, l, m} y^{m}\left(\lambda+y^{2}\right)^{-\left(\frac{m+3}{2}\right)} d y
$$

Taboga (2017, p.413, s.50.1.5) showed that (21) can be rewritten as:

$$
\mu_{g}^{\prime}=\left(1+(-1)^{g}\right) w_{i, j, k, l, m} \int_{0}^{+\infty} y^{g+m}\left(\lambda+y^{2}\right)^{-\left(\frac{m+3}{2}\right)} d y
$$

After some algebra, using the Beta function expression $B(\theta, \gamma)=\int_{0}^{+\infty} y^{\theta-1}(1+y)^{-\theta-\gamma} d y$. The $\mathrm{r}^{\text {th }}$ moment is given by

$$
\mu_{g}^{\prime}=\left\{\begin{array}{cl}
w_{i, j, k, l, m} \lambda^{\frac{g-2}{2}} \mathrm{~B}\left(\frac{g+m+1}{2}, \frac{2-g}{2}\right) & g=\text { even } \\
0 & g=\text { odd }
\end{array}\right.
$$

where $w_{i, j, k, l, m}=\alpha \lambda \frac{1}{2^{l+1}} \sum_{i, j, k, l=0}^{\infty} \sum_{m=0}^{l} \frac{(-1)^{i+j+k+l} \alpha^{i}}{i !}\left(\begin{array}{c}-(i+2) \\ j\end{array}\right)\left(\begin{array}{c}i+j \\ l\end{array}\right)\left(\begin{array}{l}k \\ l\end{array}\right)\left(\begin{array}{c}l \\ m\end{array}\right)$

The incomplete moment of the $\mathrm{OE}_{S \mathrm{ST}}$ distribution is derived. Let $Y \sim O E_{S T}(\alpha, \lambda)$ be a random variable, the $\mathrm{g}^{\text {th }}$ incomplete moment for any $t>0$ is given by

$$
\varphi_{g}^{\prime}(t)=\int_{0}^{t} y^{g} w_{i, j, k, l, m} y^{m}\left(\lambda+y^{2}\right)^{-\left(\frac{m+3}{2}\right)} d y
$$

After some algebra, using the Beta function expression $B(z, \theta, \gamma)=\int_{0}^{z} y^{\theta-1}(1-y)^{\gamma-1} d y$. The $\mathrm{r}^{\text {th }}$ incomplete moment is given by

$$
\begin{aligned}
& \varphi_{g}^{\prime}(t)=w_{i, j, k, l, m} \lambda^{\frac{g-2}{2}} \mathrm{~B}\left(t, \frac{g+m+1}{2}, \frac{2-g}{2}\right) \\
& \text { where } w_{i, j, k, l, m}=\alpha \lambda \frac{1}{2^{l+1}} \sum_{i, j, k, l=0}^{\infty} \sum_{m=0}^{l} \frac{(-1)^{i+j+k+l} \alpha^{i}}{i !}\left(\begin{array}{c}
-(i+2) \\
j
\end{array}\right)\left(\begin{array}{c}
i+j \\
l
\end{array}\right)\left(\begin{array}{l}
k \\
l
\end{array}\right)\left(\begin{array}{l}
l \\
m
\end{array}\right)
\end{aligned}
$$

Remark: The first incomplete moment $\varphi_{1}^{\prime}(t)=\int_{0}^{t} y f(y) d y$ of $\mathrm{OE}_{\mathrm{ST}}$ distribution can be obtained by inserting $g=1$ in (25).

\subsection{Characteristics Function}

The characteristics function of a random variable $Y$ is a function $\varphi_{X}(t)$ defined as: 


$$
\varphi_{X}(t)=\mathrm{E}\left(e^{i t X}\right)=\sum_{g=0}^{\infty} \frac{(i t)^{g}}{g !} \mu_{g}^{\prime}
$$

Inserting Equation (23) into Equation (26), the characteristics function of the odd exponentiated skew$\mathrm{t}\left(\mathrm{OE}_{\mathrm{ST}}\right)$ distribution is given as:

$$
\begin{aligned}
& \varphi_{X}(t)=\sum_{g=0}^{\infty} \frac{(i t)^{g}}{g !}\left\{\begin{array}{cr}
w_{i, j, k, l, m} \lambda^{\frac{g-2}{2}} \mathrm{~B}\left(\frac{g+m+1}{2}, \frac{2-g}{2}\right) & g=\text { even } \\
0 & g=\text { odd }
\end{array}\right. \\
& \text { where } w_{i, j, k, l, m}=\alpha \lambda \frac{1}{2^{l+1}} \sum_{i, j, k, l=0}^{\infty} \sum_{m=0}^{l} \frac{(-1)^{i+j+k+l} \alpha^{i}}{i !}\left(\begin{array}{c}
-(i+2) \\
j
\end{array}\right)\left(\begin{array}{c}
i+j \\
l
\end{array}\right)\left(\begin{array}{l}
k \\
l
\end{array}\right)\left(\begin{array}{l}
l \\
m
\end{array}\right)
\end{aligned}
$$

\subsection{Order Statistics}

Let $Y_{1}, Y_{2}, \ldots, Y_{n}$ be a random sample from a continuous distribution and $Y_{1: n}<Y_{2: n}<\ldots<Y_{n: n}$ are the order statistics obtained from the sample. The $\mathrm{r}^{\text {th }}$ order statistic $Y_{r: n}$ is defined as

$$
f_{r: n}(y)=\frac{1}{\mathrm{~B}(r, n-r+1)} \sum_{l=0}^{n-r}(-1)^{l}\left(\begin{array}{c}
n-r \\
l
\end{array}\right)[G(y)]^{r+l-1} g(y)
$$

Inserting Equations (5) and (6) in Equation (28), applying series expansion. The $\mathrm{r}^{\text {th }}$ order statistics for $\mathrm{OE}_{\mathrm{ST}}$ distribution is given as

$$
\begin{aligned}
& f_{r: n}(y)=\frac{1}{\mathrm{~B}(p, n-p+1)} \vartheta_{l, i, j, k, g, h, m} y^{m}\left(\lambda+y^{2}\right)^{-\left(\frac{m+3}{2}\right)} \\
& \text { where } \vartheta_{l, i, j, k, g, h, h, m}=\frac{1}{2^{h+1}} \alpha \lambda \sum_{l=0}^{n-p} \sum_{i, j, k, g, h=0}^{\infty} \sum_{m=0}^{h} \frac{(-1)^{i+j+k+l+g+h}(\alpha(i+1))^{j}}{j !}\left(\begin{array}{c}
p+l-1 \\
i
\end{array}\right)\left(\begin{array}{c}
-(j+2) \\
k
\end{array}\right)\left(\begin{array}{c}
n-p \\
l
\end{array}\right)\left(\begin{array}{c}
j+k \\
g
\end{array}\right)\left(\begin{array}{c}
g \\
h
\end{array}\right)\left(\begin{array}{c}
h \\
m
\end{array}\right)
\end{aligned}
$$

Remark: The minimum and maximum order statistics is derived by setting $r=1$ and $r=n$ in Equation (29).

\subsection{Entropies}

The variation of uncertainty in a random variable is normally measured by the entropy (Rényi, 1961). The Rényi entropy $\mathrm{I}_{R(\delta)}$ is expressed as:

$$
\mathrm{I}_{R(\delta)}=\frac{1}{1-\delta} \log \int_{-\infty}^{+\infty} f(y)^{\delta} d y, \quad \delta>0 \text { and } \delta \neq 1
$$

Using the PDF mixture representation of $\mathrm{OE}_{\mathrm{ST}}$ distribution in (19), $f(y)^{\delta}$ is given as: 
$f(y)^{\delta}=w_{i, j, k, l, m} y^{m}\left(\lambda+y^{2}\right)^{-(m+3 \delta / 2)}$

where $w_{i, j, k, l, m}=\frac{(\alpha \lambda)^{\delta}}{2^{l+\delta}} \sum_{i, j, k, l=0}^{\infty} \sum_{m=0}^{l} \frac{(-1)^{i+j+k+l}(\alpha \delta)^{i}}{i !}\left(\begin{array}{c}-(i+2 \delta) \\ j\end{array}\right)\left(\begin{array}{c}i+j \\ k\end{array}\right)\left(\begin{array}{l}k \\ l\end{array}\right)\left(\begin{array}{c}l \\ m\end{array}\right)$

Hence, the Rényi entropy of the $\mathrm{OE}_{S \mathrm{ST}}$ distribution using the expression by Taboga (2017, p.413, s.50.1.5), is expressed as:

$$
\mathrm{I}_{R(\delta)}=\frac{1}{1-\delta} \log \left(\left(1+(-1)^{g}\right) w_{i, j, k, l, m} \int_{0}^{+\infty} y^{m}\left(\lambda+y^{2}\right)^{-(m+3 \delta / 2)} d y\right)
$$

Using the expression of the Beta function $B(\theta, \gamma)=\int_{0}^{+\infty} y^{\theta-1}(1+y)^{-\theta-\gamma} d y$. The Rényi entropy of the $\mathrm{OE}_{\text {ST }}$ distribution is given as:

$$
\mathrm{I}_{R(\delta)}=\frac{1}{1-\delta} \log \left\{\begin{array}{cc}
w_{i, j, k, l, m} \lambda^{\frac{1-3 \delta}{2}} \mathrm{~B}\left(\frac{m+1}{2}, \frac{3 \delta-1}{2}\right) & g=\text { even } \\
0 & g=\text { odd }
\end{array}\right.
$$

Likewise, the q-entropy (Tsallis, 1988) is defined as:

$$
\mathrm{H}_{q}=\frac{1}{q-1} \log \left(1-\int_{-\infty}^{+\infty} f(y)^{q} d y\right), q>0 \text { and } q \neq 0
$$

Using the PDF mixture representation of $\mathrm{OE}_{\mathrm{ST}}$ distribution in (19), $f(y)^{q}$ is given as:

$$
f(y)^{q}=w_{i, j, k, l, m} y^{m}\left(\lambda+y^{2}\right)^{-(m+3 q / 2)}
$$

where $w_{i, j, k, l, m}=\frac{(\alpha \lambda)^{q}}{2^{l+q}} \sum_{i, j, k, l=0}^{\infty} \sum_{m=0}^{l} \frac{(-1)^{i+j+k+l}(\alpha q)^{i}}{i !}\left(\begin{array}{c}-(i+2 q) \\ j\end{array}\right)\left(\begin{array}{c}i+j \\ k\end{array}\right)\left(\begin{array}{l}k \\ l\end{array}\right)\left(\begin{array}{l}l \\ m\end{array}\right)$

Hence, the q-entropy of the $\mathrm{OE}_{S T}$ distribution is given as:

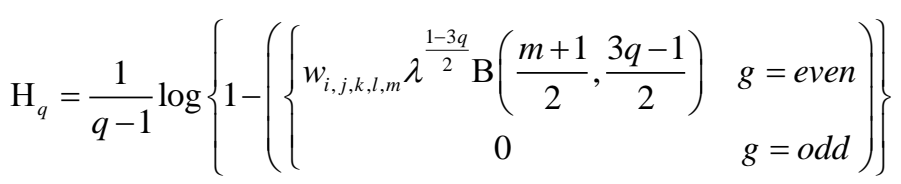

\section{MODEL ESTIMATION}

\subsection{Parameters Estimation}

Let $Y_{1}, Y_{2}, \ldots, Y_{n}$ be a random sample from the $\mathrm{OE}_{\text {ST }}$ distribution with unknown parameter vector $v=(\alpha, \lambda)^{T}$. The likelihood $(L)$ of the $\mathrm{OE}_{\mathrm{ST}}$ distribution is expressed as: 


$$
L(v)=\prod_{i=1}^{n}\left(\frac{\alpha \lambda}{2\left(\lambda+y^{2}\right)^{3 / 2}\left[1-\left(\frac{1}{2}\left(1+\frac{y}{\sqrt{\lambda+y^{2}}}\right)\right)\right]^{2}} e^{\left.-\alpha \frac{\left(\frac{1}{2}\left(1+\frac{y}{\sqrt{\lambda+y^{2}}}\right)\right)}{1-\left(\frac{1}{2}\left(1+\frac{y}{\sqrt{\lambda+y^{2}}}\right)\right)}\right)}\right.
$$

The log-likelihood function $(L L)$ is given as:

$$
\begin{aligned}
L L=\log L(v)=n \log \alpha+n \log \lambda-n \log 2-3 / 2 \sum_{i=1}^{n} \log \left(\lambda+y^{2}\right)-\alpha \sum_{i=1}^{n} \frac{\left(\frac{1}{2}\left(1+\frac{y}{\sqrt{\lambda+y^{2}}}\right)\right)}{1-\left(\frac{1}{2}\left(1+\frac{x}{\sqrt{\lambda+y^{2}}}\right)\right)} \\
\quad-2 \sum_{i=1}^{n} \log \left(1-\frac{1}{2}\left(1+\frac{y}{\sqrt{\lambda+y^{2}}}\right)\right)
\end{aligned}
$$

Taking the partial derivative of the $\log$-likelihood $l$, with respect to $\alpha$ and $\lambda$ equating to zero, the normal equations are obtained as follows:

$$
\frac{\partial L L}{\partial \alpha}=\frac{n}{\alpha}-\sum_{i=1}^{n} \frac{\left(\frac{1}{2}\left(1+\frac{x}{\sqrt{\lambda+y^{2}}}\right)\right)}{1-\left(\frac{1}{2}\left(1+\frac{x}{\sqrt{\lambda+y^{2}}}\right)\right)}=0
$$

$$
\frac{\partial L L}{\partial \lambda}=\frac{n}{\lambda}-\frac{3}{2} \sum_{i=1}^{n} \frac{1}{\left(\lambda+y^{2}\right)}+\alpha \sum_{i=1}^{n} \frac{y}{\sqrt{\lambda+y^{2}}\left(\sqrt{\lambda+y^{2}}-y\right)^{2}}+\sum_{i=1}^{n} \frac{y}{\left(\lambda+y^{2}\right)\left(-\sqrt{\lambda+y^{2}}+y\right)}=0
$$

The non-linear equations (38) and (39) are solved numerically via iterative methods using statistical software such as R, MATLAB and Maple. The maximum likelihood estimates (MLEs) are asymptotic normally distributed i.e., $\sqrt{n}(\hat{\alpha}-\alpha, \hat{\lambda}-\lambda)$ follow $\mathrm{N}_{2}(0, \Sigma)$, where $\Sigma$ is the variance-covariance matrix obtained by inverting the observed Fisher information $(F)$ given as follows:

$$
F=\left[\begin{array}{cc}
\frac{\partial^{2} l}{\partial \alpha^{2}} & \frac{\partial^{2} l}{\partial \alpha \partial \lambda} \\
\frac{\partial^{2} l}{\partial \alpha \partial \lambda} & \frac{\partial^{2} l}{\partial \lambda^{2}}
\end{array}\right]
$$

For each parameter of $\mathrm{OE}_{\mathrm{ST}}$ distribution, the asymptotic $(1-\tau) 100 \%$ confidence intervals are estimated with 
$\hat{\alpha} \pm Z_{\tau / 2} \sqrt{\Sigma_{11}}$

$\hat{\lambda} \pm Z_{\tau / 2} \sqrt{\Sigma_{22}}$

where, upper $\tau^{\text {th }}$ percentile of the standard normal distribution is $\mathrm{Z}_{\tau}$.

\subsection{Simulations Study}

The simulation study for the average MLEs, absolute bias, variance, Mean Square Errors (MSE), and Root Mean Square Errors (RMSE) are performed for the OE $\mathrm{E}_{\mathrm{ST}}$ distribution. For $N=10000$, random numbers of sample size $n=30,50,150,300,1000$ are generated using the $\mathrm{OE}_{\mathrm{ST}}$ quantile function as in equation (9). The absolute bias, MSE and RMSE are computed for $\hat{S}=(\hat{\alpha}, \hat{\lambda})$ using

$$
\operatorname{Absias}_{s}=\left|\frac{1}{N} \sum_{i=1}^{N}\left(\hat{S}_{i}-S\right)\right|, \quad \quad M S E_{s}=\frac{1}{N} \sum_{i=1}^{N}\left(\hat{S}_{i}-S\right)^{2}, \quad R M E_{s}=\sqrt{\frac{1}{N} \sum_{i=1}^{N}\left(\hat{S}_{i}-S\right)^{2}}
$$

The simulation results for the average MLEs, absolute bias, variance, MSEs, and RMSEs for different combinations of the parameters $\alpha$ and $\lambda$ are given in Table 2. These estimates are sensibly consistent and approach the parameter values as the sample size increases. The absolute bias, variance, RMSEs, and MSEs decrease for all parameter mixtures as the sample size increases which implies that the $\mathrm{OE}_{\text {Sт }}$ parameter estimates are very much consistent, better estimated and approaches the arbitrary selected parameter values as the sample size increases.

Table 2: Simulation results

\begin{tabular}{|c|c|c|c|c|c|c|}
\hline \multicolumn{7}{|c|}{$(\alpha=1.2, \lambda=0.7)$} \\
\hline $\mathbf{n}$ & Par & AE & ABS & Var & MSE & RMSE \\
\hline $\mathbf{3 0}$ & $\alpha$ & 1.241 & 0.041 & 0.064 & 0.066 & 0.256 \\
\hline \multirow{2}{*}{$\mathbf{5 0}$} & $\lambda$ & 0.687 & 0.013 & 0.061 & 0.061 & 0.248 \\
\hline & $\lambda$ & 1.222 & 0.022 & 0.035 & 0.035 & 0.187 \\
\hline $\mathbf{1 5 0}$ & $\alpha$ & 0.691 & 0.009 & 0.037 & 0.037 & 0.193 \\
\hline \multirow{3}{*}{$\mathbf{3 0 0}$} & $\lambda$ & 0.696 & 0.004 & 0.012 & 0.012 & 0.110 \\
\hline & $\alpha$ & 1.203 & 0.003 & 0.005 & 0.005 & 0.072 \\
\hline $\mathbf{1 0 0 0}$ & $\alpha$ & 0.698 & 0.002 & 0.006 & 0.006 & 0.077 \\
\hline & $\lambda$ & 1.201 & 0.000 & 0.001 & 0.001 & 0.039 \\
\hline
\end{tabular}




\begin{tabular}{|c|c|c|c|c|c|c|}
\hline \multicolumn{7}{|c|}{$(\alpha=1.5, \lambda=1.0)$} \\
\hline $\mathbf{n}$ & Par & AE & ABS & Var & MSE & RMSE \\
\hline $\mathbf{3 0}$ & $\alpha$ & 1.570 & 0.070 & 0.105 & 0.110 & 0.332 \\
\hline & $\lambda$ & 0.978 & 0.022 & 0.130 & 0.131 & 0.362 \\
\hline $\mathbf{5 0}$ & $\alpha$ & 1.539 & 0.039 & 0.055 & 0.056 & 0.237 \\
\hline & $\lambda$ & 0.985 & 0.015 & 0.079 & 0.079 & 0.281 \\
\hline $\mathbf{1 5 0}$ & $\alpha$ & 1.510 & 0.101 & 0.016 & 0.016 & 0.127 \\
\hline \multirow{3}{*}{$\mathbf{3 0 0}$} & $\lambda$ & 0.994 & 0.006 & 0.025 & 0.025 & 0.160 \\
\hline & $\alpha$ & 1.506 & 0.006 & 0.008 & 0.008 & 0.088 \\
\hline $\mathbf{1 0 0 0}$ & $\alpha$ & 0.997 & 0.003 & 0.012 & 0.012 & 0.112 \\
\hline & $\lambda$ & 1.501 & 0.001 & 0.002 & 0.002 & 0.048 \\
\hline
\end{tabular}

\begin{tabular}{|c|c|c|c|c|c|c|}
\hline \multicolumn{7}{|c|}{$(\alpha=1.7, \lambda=1.2)$} \\
\hline $\mathbf{n}$ & Par & AE & ABS & Var & MSE & RMSE \\
\hline $\mathbf{3 0}$ & $\alpha$ & 1.795 & 0.095 & 0.147 & 0.156 & 0.395 \\
\hline \multirow{2}{*}{$\mathbf{5 0}$} & $\lambda$ & 1.170 & 0.030 & 0.195 & 0.195 & 0.442 \\
\hline & $\lambda$ & 1.752 & 0.052 & 0.074 & 0.077 & 0.277 \\
\hline $\mathbf{1 5 0}$ & $\alpha$ & 1.180 & 0.020 & 0.118 & 0.118 & 0.344 \\
\hline \multirow{3}{*}{$\mathbf{3 0 0}$} & $\lambda$ & 1.714 & 0.014 & 0.021 & 0.021 & 0.146 \\
\hline & $\alpha$ & 1.192 & 0.007 & 0.038 & 0.038 & 0.195 \\
\hline $\mathbf{1 0 0 0}$ & $\alpha$ & 1.196 & 0.004 & 0.019 & 0.019 & 0.136 \\
\hline & $\lambda$ & 1.702 & 0.002 & 0.003 & 0.003 & 0.055 \\
\hline
\end{tabular}




\begin{tabular}{|c|c|c|c|c|c|c|}
\hline \multicolumn{7}{|c|}{$(\alpha=2.0, \lambda=1.5)$} \\
\hline $\mathbf{n}$ & Par & AE & ABS & Var & MSE & RMSE \\
\hline $\mathbf{3 0}$ & $\alpha$ & 2.141 & 0.141 & 0.244 & 0.264 & 0.514 \\
\hline & $\lambda$ & 1.457 & 0.043 & 0.324 & 0.326 & 0.571 \\
\hline $\mathbf{5 0}$ & $\alpha$ & 2.078 & 0.078 & 0.115 & 0.121 & 0.348 \\
\hline & $\lambda$ & 1.471 & 0.029 & 0.195 & 0.196 & 0.443 \\
\hline $\mathbf{1 5 0}$ & $\alpha$ & 2.022 & 0.022 & 0.031 & 0.031 & 0.177 \\
\hline \multirow{3}{*}{$\mathbf{3 0 0}$} & $\lambda$ & 1.489 & 0.010 & 0.063 & 0.063 & 0.251 \\
\hline & $\alpha$ & 2.011 & 0.011 & 0.014 & 0.015 & 0.121 \\
\hline $\mathbf{1 0 0 0}$ & $\alpha$ & 1.495 & 0.005 & 0.031 & 0.031 & 0.176 \\
\hline & $\lambda$ & 2.003 & 0.003 & 0.004 & 0.004 & 0.066 \\
\hline
\end{tabular}

\section{$5 \quad$ APPLICATION}

In this section, the flexibility and superiority of the odd exponentiated skew-t $\left(\mathrm{OE}_{\mathrm{ST}}\right)$ distribution over other two-parameter distributions are demonstrated using a real dataset. The odd exponentiated skew-t (OE $\mathrm{OET}_{\mathrm{ST}}$ distribution is compared with other competitive distributions such as the type-I half logistic skew-t (TIHLST), half logistic skew-t (HLST), Fréchet (FT), Pareto (PE), Lomax (LOMX), inverse Pareto (INVPE), type-I half logistic Burr X (TIHLBX) and skew Student-t (ST). The descriptive statistics of the dataset are provided in Table 3.

The dataset represents the survival times (in days) of 72 guinea pigs infected with virulent tubercle bacilli. The dataset is given as follows:

$0.1,0.33,0.44,0.56,0.59,0.72,0.74,0.77,0.92,0.93,0.96,1,1,1.02,1.05,1.07,1.07,1.08,1.08$, $1.08,1.09,1.12,1.13,1.15,1.16,1.2,1.21,1.22,1.22,1.24,1.3,1.34,1.36,1.39,1.44,1.46,1.53$, $1.59,1.6,1.63,1.63,1.68,1.71,1.72,1.76,1.83,1.95,1.96,1.97,2.02,2.13,2.15,2.16,2.22,2.3$, $2.15,2.16,2.22,2.3,2.31,2.4,2.45,2.51,2.53,2.54,2.54,2.78,2.93,3.27,3.42,3.47,3.61,4.02$, 4.32, 4.58, 5.55. This dataset has previously been used by Jamal et al. (2019), Umar et al. (2019), Leren and Abdullahi (2020) and Ampadu and Anafo (2019).

Table 3: Descriptive statistics of the dataset.

\begin{tabular}{|l|l|l|l|l|l|l|}
\hline & $\mathbf{n}$ & Mean & Median & SD & Skewness & Kurtosis \\
\hline Dataset & 72 & 1.791 & 1.595 & 1.011 & 1.294 & 5.046 \\
\hline
\end{tabular}

The performance measures are applied using the R-software package "AdequacyModel" to evaluate the fit of the distributions specified above. The distribution parameters are estimated using the 
maximum likelihood estimation procedure. The following performance measures: Hannan-Quinn information criterion (HQIC), Akaike Information Criterion (AIC), Bayesian Information Criterion (BIC), Anderson Darling (AD), Cramer-von Mises (CVM), Kolmogorov-Smirnov (K-S) statistic and its p-value are provided in Table 4. The distribution is of a good fit if all the performance measures are smaller and the p-value is larger. Lastly, Table 5 presents the $95 \%$ and $99 \%$ confidence intervals for the $\mathrm{OE}_{\mathrm{ST}}$ distribution parameters.

Table 4: MLEs (SE) and performance measures.

\begin{tabular}{|c|c|c|c|c|c|c|c|c|c|}
\hline Model & Par & MLEs (SEs) & AIC & BIC & HQIC & CVM & AD & K-S & $\begin{array}{c}\text { p- } \\
\text { value }\end{array}$ \\
\hline \multirow{2}{*}{$\mathrm{OE}_{\mathrm{ST}}$} & $\hat{\alpha}$ & $0.0054(0.0022)$ & \multirow{2}{*}{206.52} & \multirow{2}{*}{211.18} & \multirow{2}{*}{208.28} & \multirow{2}{*}{0.054} & \multirow{2}{*}{0.278} & \multirow{2}{*}{0.085} & \multirow{2}{*}{0.645} \\
\hline & $\hat{\lambda}$ & $0.0919(0.0397)$ & & & & & & & \\
\hline \multirow{2}{*}{ TIHLST } & $\hat{\alpha}$ & $0.6579(0.0889)$ & \multirow{2}{*}{288.94} & \multirow{2}{*}{293.60} & \multirow{2}{*}{290.81} & \multirow{2}{*}{0.064} & \multirow{2}{*}{0.366} & \multirow{2}{*}{0.374} & \multirow{2}{*}{$\begin{array}{c}1.2 \mathrm{e}- \\
09\end{array}$} \\
\hline & $\hat{\lambda}$ & $1.5812(0.5509)$ & & & & & & & \\
\hline HLST & $\hat{\lambda}$ & $3.5701(0.7450)$ & 297.13 & 299.46 & 298.06 & 0.099 & 0.579 & 0.456 & $\begin{array}{c}3.8 \mathrm{e}- \\
14\end{array}$ \\
\hline \multirow{2}{*}{ FT } & $\hat{\alpha}$ & $1.1771(0.0819)$ & \multirow[b]{2}{*}{255.59} & \multirow{2}{*}{260.25} & \multirow{2}{*}{257.45} & \multirow{2}{*}{0.575} & \multirow[b]{2}{*}{3.659} & \multirow{2}{*}{0.192} & \multirow[b]{2}{*}{0.001} \\
\hline & $\hat{\lambda}$ & $1.0846(0.1125)$ & & & & & & & \\
\hline \multirow{3}{*}{ PE } & $\hat{\alpha}$ & $47.126(44.788)$ & \multirow{3}{*}{245.71} & & & & & & \\
\hline & $\hat{\theta}$ & $84.038(81.125)$ & & 250.38 & 247.58 & 0.078 & 0.524 & 0.296 & $3.3 \mathrm{e}-$ \\
\hline & $\hat{\beta}$ & $93.3635(73.8610)$ & & & & & & & \\
\hline & $\hat{\alpha}$ & $40.0722(51.3010)$ & & & & & & & $2.3 \mathrm{e}-$ \\
\hline LOMX & $\hat{\beta}$ & $0.0142(0.0183)$ & 245.92 & 250.58 & 247.78 & 0.078 & 0.522 & 0.300 & 06 \\
\hline & $\hat{\alpha}$ & $13.0025(11.3630)$ & & & & & & & $8.6 \mathrm{e}-$ \\
\hline INVPE & $\hat{\beta}$ & $0.0980(0.0914)$ & 258.46 & 263.12 & 260.32 & 0.320 & 2.160 & 0.257 & 05 \\
\hline & $\hat{\alpha}$ & $-0.0788(0.0859)$ & & & & & & & \\
\hline TIHLBX & $\hat{\theta}$ & $0.7835(0.0762)$ & 209.059 & 216.05 & 211.85 & 0.164 & 1.030 & 0.104 & 0.377 \\
\hline & $\hat{\lambda}$ & $25.8169(45.9911)$ & & & & & & & \\
\hline ST & $\hat{\lambda}$ & $5.7271(1.2579)$ & 346.19 & 348.52 & 347.12 & 0.117 & 0.698 & 0.578 & $\begin{array}{c}2.2 \mathrm{e}- \\
16\end{array}$ \\
\hline
\end{tabular}


Histogram and theoretical densiti

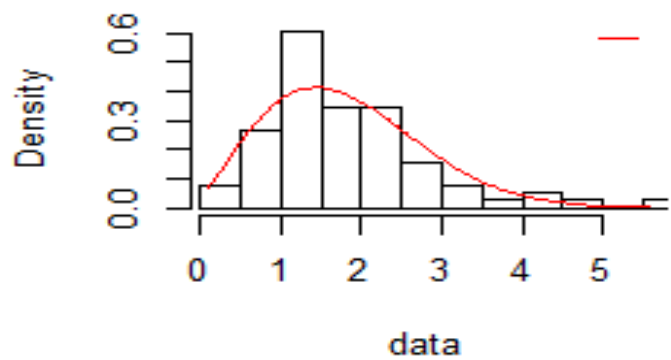

P-P plot

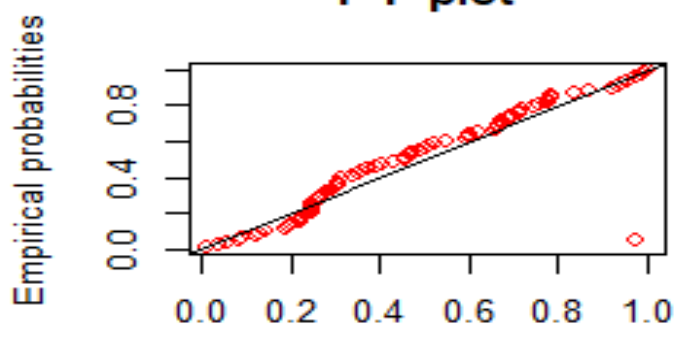

Theoretical probabilities

\section{Empirical and theoretical CDFs}

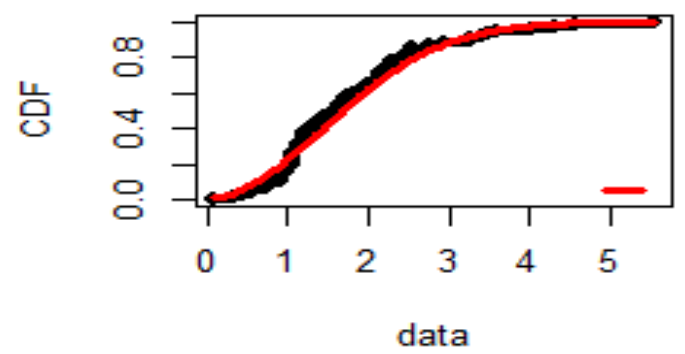

Q-Q plot

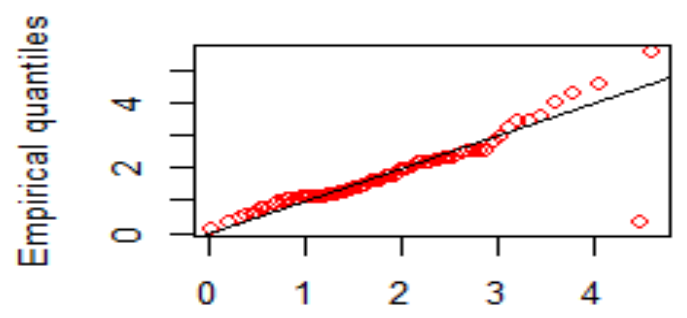

Theoretical quantiles

Figure 4: Fitted density function plot (top left panel), distribution function plot (top right panel), probability-probability (PP) plot (bottom left panel) and quantile-quantile (QQ) plot (bottom right panel) of the $\mathrm{OE}_{\mathrm{ST}}$ distribution.
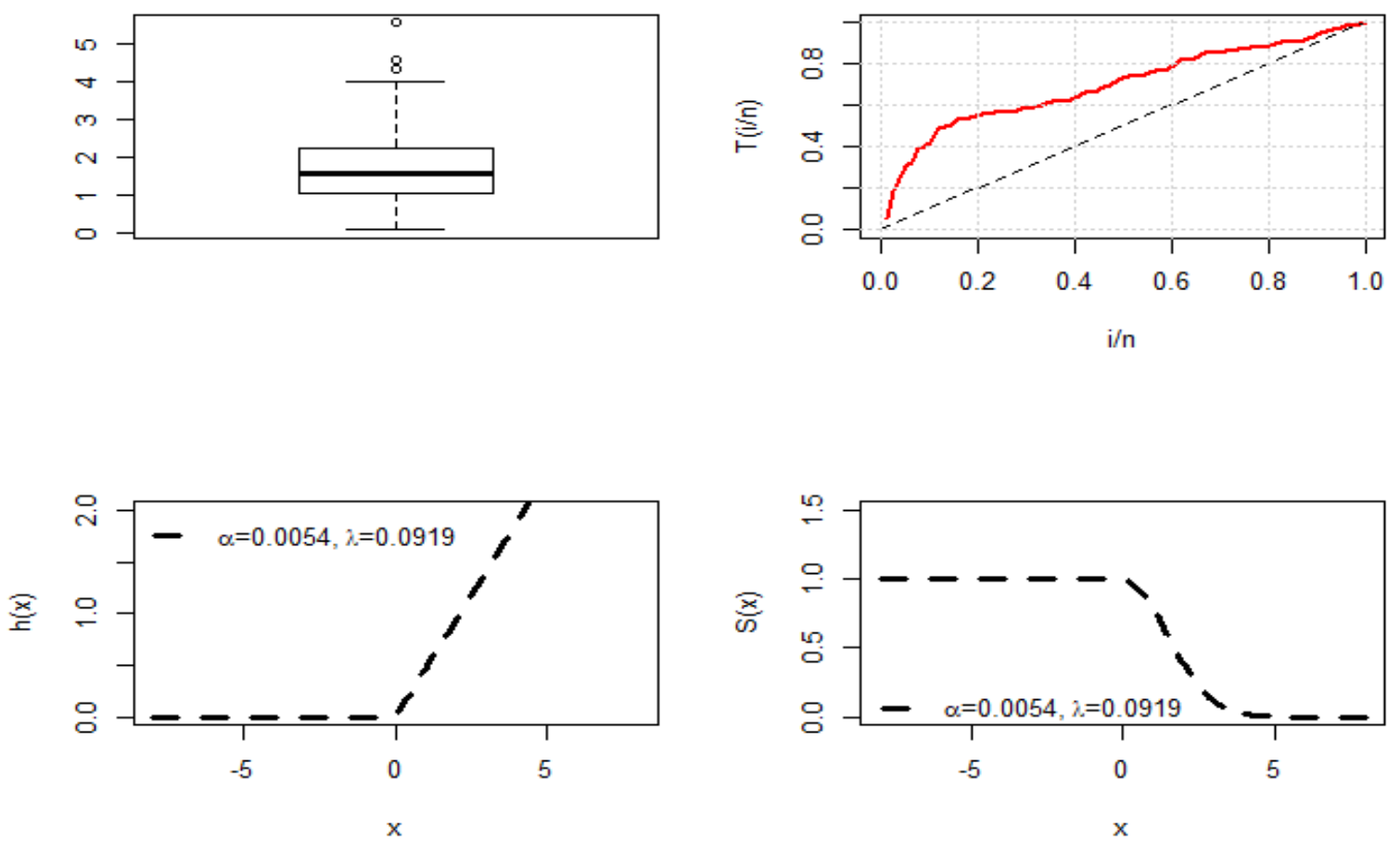
Figure 5: The Box plot (top left panel), total time of test (TTT) plot (top right panel), $\mathrm{OE}_{S \mathrm{ST}}$ hazard rate function plot (bottom left panel) and $\mathrm{OE}_{\mathrm{ST}}$ survival function plot (bottom right panel).

From the results in Table 4, the performance measures of the $\mathrm{OE}_{\mathrm{ST}}$ distribution are smaller when compared to other fitted distributions, so we infer that the $\mathrm{OE}_{\mathrm{ST}}$ distribution provides a good fit than the other distributions. The flexibility and fitness of the $\mathrm{OE}_{\mathrm{ST}}$ distribution is visible from Figure 4. It is clear that $\mathrm{OE}_{\mathrm{ST}}$ distribution provides an appropriate fit for the dataset based on the density function, distribution function, P-P plot and Q-Q plot in Figure 4. The TTT (total time on test) plot in Figure 5, shows that the dataset exhibits an increasing failure rate function and $\mathrm{OE}_{\mathrm{ST}}$ is capable of accommodating increasing failure rates. Likewise, the Box plot of the dataset is shown in Figures 5.

Furthermore, the hazard rate and survival plots of the $\mathrm{OE}_{\text {ST }}$ distribution, using the parameter estimates in Table 4 are also depicted in Figure 5. The hazard rate shape based on the OE estimates is increasing and $\mathrm{J}$-shaped. The J-shaped means that the $\mathrm{OE}_{\mathrm{ST}}$ distribution tend to have some observations at one end, very few in the middle and a large number of observations at the other end which gives it the capability of handling skewed and heavy tail datasets. The results in Table 5, shows that the parameter estimates fall within the $95 \%$ and $99 \%$ confidence intervals.

Table 5. $\mathrm{OE}_{\mathrm{ST}}$ distribution parameter estimates confidence intervals

\begin{tabular}{|l|ll|l|}
\hline $\mathbf{C I}$ & $\alpha$ & $\lambda$ \\
\hline $95 \%$ & {$\left[\begin{array}{lll}0.00109 & 0.00971\end{array}\right]$} & {$\left[\begin{array}{ll}0.01409 & 0.16971\end{array}\right]$} \\
\hline $99 \%$ & {$\left[\begin{array}{ll}-0.00025 & 0.01105\end{array}\right]$} & {$\left[\begin{array}{lll}-0.01013 & 0.19393\end{array}\right]$} \\
\hline
\end{tabular}

\section{CONCLUSION}

This article presents a new two-parameter distribution known as the odd exponentiated skew-t (OEST) distribution using the odd exponentiated transformation. The flexibility of the skew-t distribution is improved using this transformation. This mixture representation is important to derive several structural properties of this distribution in full generality. Some of them are provided such as the ordinary and incomplete moments, quantile function, entropy, characteristic function and order statistics. The new distribution parameter estimates are derived using the maximum likelihood estimation (MLE) procedure and simulation study showed that the MLE performed well in estimating the parameters of the new distribution. The application using a real dataset indicates that the $\mathrm{OE}_{\mathrm{ST}}$ distribution outperformed the other competing distributions. Future research study will compare the performance of the $\mathrm{OE}_{S \mathrm{ST}}$ error conditional distribution to existing error conditional distributions such as the normal distribution, Student-t distribution, generalized error distribution, and its skew variants in modeling and forecasting volatility using GARCH framework. 


\section{CONFLICTS OF INTEREST}

Authors declare that there are no conflicts of interest.

\section{REFERENCES}

Aas K., and Haff I. H. (2006). The Generalised Hyperbolic Skew Student's t-distribution. Journal of Financial Econometric, 4(2), 275-309.

Ampadu C. B. and Anafo A. Y. (2019). Maximum likelihood estimation in an alpha-power. Annals of Biostatistics and Biometric applications, 2(4), 1-5.

Azzalini, A. and Capitanio, A. (2003). Distributions generated by perturbation of symmetry with emphasis on a multivariate skew-t distribution. J. Roy. Statist. Soc., B, 65, 367-389.

Azzalini, A. and Capitanio, A (2014). The Skew-Normal and Related Families. IMS monographs. Cambridge University Press.

Basalamah D., Ning W., and Gupta A. (2018). The beta skew-t distribution and its properties. Journal of Statistical theory and practice, 12, 837-860.

Bourguignon, M., Silva R. B. and Cordeiro G. M. (2014). The Weibull-G family of probability distributions. J. Data Sci. 12, 53-68.

Gupta A. K., Chang, F. C. and Huang, W. J. (2002). Some skew symmetric models. Random Oper. and Stoch. Eqn., 10, 133-140.

Jamal F., Reyad H. M., Nasir M. A., Chesneau C., Shah M. A. A., Ahmed S. O. (2019). Topp-Leone Weibull-Lomax distribution: Properties, Regression Model and Applications. 2019. ffhal02270561 .

Johnson, N. L., Kotz, S. and Balakrishnan, N. (1995). Continuous Univariate Distributions, vol. 2, 2nd edn. New York: Wiley.

Jones M. C. (2004). Families of distributions of order statistics. Statistical Methodology, 6, 70-91.

Jones, M. C. (2001). A skew t distribution. In Probability and Statistical Models with Applications (eds C. A. Charalambides, M. V. Koutras and N. Balakrishnan), pp. 269-277. London: Chapman and Hall.

Jones, M. C. (2002). Student's simplest distribution. The Statistician, 51(1), 41-49.

Jones, M. C. and M. J. Faddy (2003). A skew extension of the t distribution, with applications. J. Roy. Statist. Soc., Ser. B 65 (2), 159-174.

Kenney, J. F. and Keeping, E. (1962). Mathematics of Statistics. D. Van Nostr and Company.

Khamis K. S., Basalamah D., Ning W., and Gupta A. (2017). The Kumaraswamy Skew-t Distribution and Its Related Properties. Communications in Statistics - Simulation and Computation, DOI: 10.1080/03610918.2017.1346801.

Leren T. G. and Abdullahi J. (2020). A transmuted Normal distribution: properties and applications. Equity journal of science and technology, 7(1), 16-34. 
Moor J. J. (1988). A quantile alternative for Kurtosis. The Statistician, 37, 25-32.

Rényi A. (1961). Proceeding of the fourth Berkeley symposium on mathematical statistics and probabilities. First Edition, University of California Press Berkeley.

Sahu, S. K., D. K. Dey, and M. D. Branco (2003). A new class of multivariate skew distributions with applications to Bayesian regression models. The Canadian Journal of Statistics 31, 129-150.

Shafiei S. and Doostparast, M. (2014). Balakrishnan skew-t distribution and associated statistical characteristics. Comm. Statist. Theory Methods, 43, 4109-4122.

Shittu O. I., Adepoju K. A. and Adeniji O. E. (2014). On the Beta Skew-t distribution in modelling stock return in Nigeria. International Journal of Modern Mathematical Sciences. 11 (2), 94-102.

Taboga, M. (2017). Lectures on probability theory and mathematical statistics. $3^{\text {rd }}$ Edn. John Wiley \& Sons, New York

Umar A. A., Eraikhuemen, I. B., Koleoso P. O., Joel J. and Ieren T. G. (2019). On the Properties and Applications of a Transmuted Lindley-Exponential Distribution. Asian Journal of Probability and Statistics, 5(3),1-13.

Tsallis C. (1988). Possible generalization of Boltzmann-Gibbs statistics. Journal of statistical Physics. 52(1-2), 479-487. 